Article

\title{
Recent Progress in Understanding the Large Scale Jets of Powerful Quasars
}

\author{
Markos Georganopoulos ${ }^{1, *}$, Eileen T. Meyer ${ }^{1}$ and Eric S. Perlman ${ }^{2}$ \\ 1 Department of Physics, University of Maryland, Baltimore, MD 21250, USA; meyer@umbc.edu \\ 2 Department of Physics, Florida Institute of Technology, Melbourne, FL 32901, USA; eperlman@fit.edu \\ * Correspondence: georgano@umbc.edu
}

Academic Editors: Jose L. Gómez, Alan P. Marscher and Svetlana G. Jorstad

Received: 7 September 2016; Accepted: 10 November 2016; Published: 23 November 2016

\begin{abstract}
Our understanding of the physics of kpc-scale quasar jets had seemed to converge to a paradigm in which these jets are as highly relativistic on the kpc scale as they are on sub-pc scales close to the central black hole. Retaining bulk Lorentz factors $(\Gamma)$ on the order of 10-20 at these distances implies a jet power comparable to or higher than their Eddington luminosity. We recently started challenging this paradigm, which was put in place to explain the surprisingly bright X-ray emission of the knots of many quasar jets as inverse Compton scattering off the cosmic microwave background $(\mathrm{IC} / \mathrm{CMB})$. We have shown that the knot X-ray emission of the archetypical jets 3C 273 and PKS 0637-752 is not due to IC/CMB. With IC/CMB disfavored, an alternative interpretation for the $\mathrm{X}$-rays is synchrotron radiation from a second population of electrons accelerated in situ up to $\sim 100 \mathrm{TeV}$. These results are the first step towards resolving the long-standing issue of the nature of the $\mathrm{X}$-ray emission in powerful quasar jets. Comprehensive observational and theoretical work on essentially all X-ray-detected large-scale quasar jets to test the IC/CMB model over a much larger population needs to be done to examine the implications of slower jets that are extremely efficient accelerators. A fascinating case can be made that-contrary to popular belief-the total radiative power of the large-scale jet of these sources is comparable to that of the quasar core. Even more so, the angle-integrated $\mathrm{TeV}$ output of these (previously thought $\mathrm{TeV}$-quiet) quasar jets likely makes them the dominant class among active galactic nuclei (AGN), exceeding the $\mathrm{TeV}$ production of so-called TeV blazars.
\end{abstract}

Keywords: active galaxies; jets; non-thermal emission mechanisms

\section{Introduction}

In active galactic nuclei (AGN), relativistic jets transport energy and mass from the sub-parsec central regions to Mpc-scale lobes, with a kinetic power comparable to that of the host galaxy and the AGN, profoundly influencing the evolution of the host, nearby galaxies, and the surrounding interstellar and intracluster medium [1,2]. The generation of these flows is tied to the process of accretion onto rotating black holes, where the magneto-rotational instability can couple the black hole's spin and magnetic field to produce high-latitude outflows close to the speed of light [3]. AGN outflows-including jets-are thus essential parts of galaxy evolution models extending back to the earliest times.

Over the last 15 years, Chandra has detected X-ray emission from more than 100 jets (see [4,5] for reviews). These exciting observations have revealed that $\mathrm{X}$-ray jets extend up to hundreds of kpc and are dominated by emission from bright jet knots and terminal hotspots. In low-power FR I jets, the optical/near-IR and X-ray fluxes fit on an extrapolation of the radio spectra (e.g., [6-8]), consistent with a single-population synchrotron origin for the emission all the way from radio through $\mathrm{X}$-ray energies. However, more powerful FR II (quasar) jets have very different spectral energy distributions 
(SEDs), with the observed X-rays being harder and at a higher flux than expected from extrapolating the radio-to-optical synchrotron spectrum, suggesting that they are part of a separate spectral component (Figure 1b). A synchrotron self-Compton (SSC) nature for the X-rays was disfavored [9,10], as the X-rays are at least a factor of 100 higher than the anticipated SSC flux (e.g., [9-13]). A controversy with serious implications for jet physics emerged over the nature of the $\mathrm{X}$-rays.
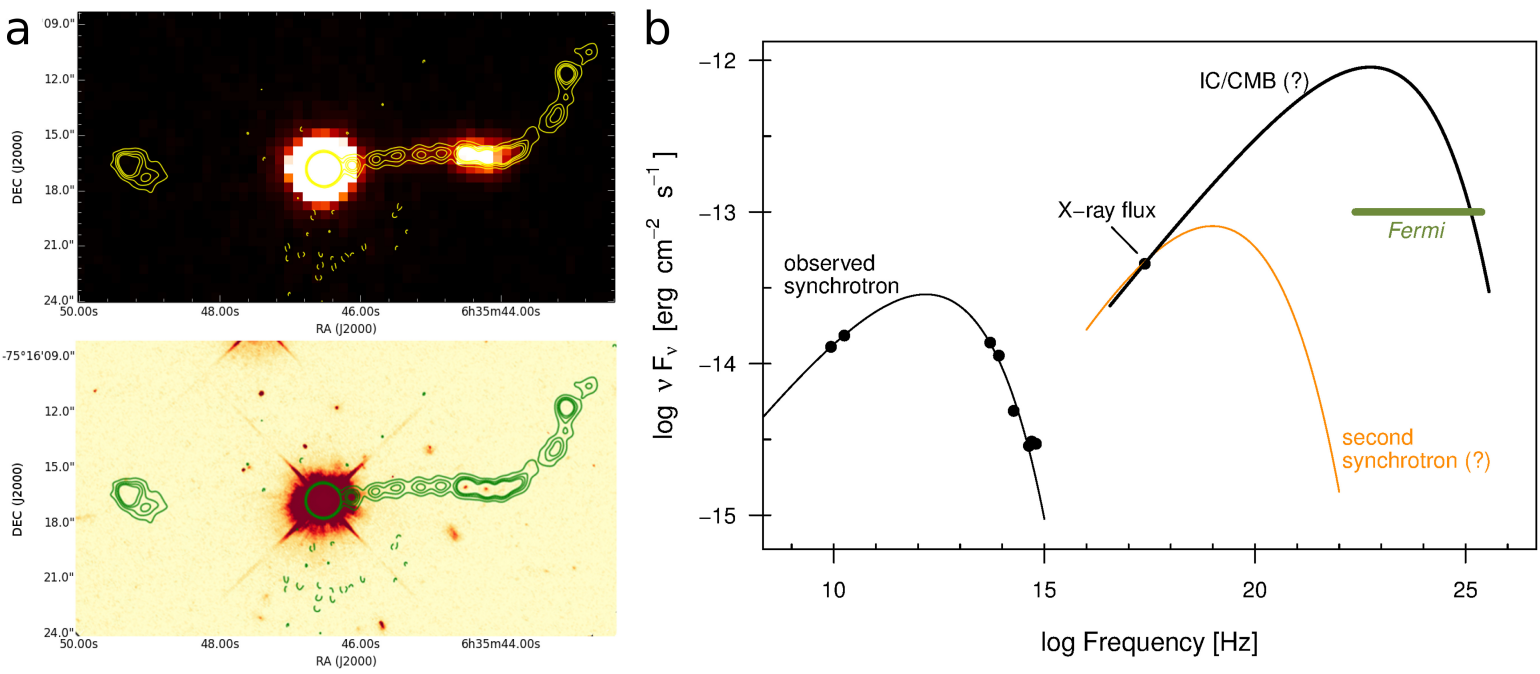

Figure 1. (a) X-ray and optical image (upper and lower panels, respectively) of the 100-kpc long jet of the powerful radio quasar PKS 0637-752, with $17 \mathrm{GHz}$ radio contours overlaid. The unexpected $\mathrm{X}$-ray detection of the jet (separately from the core) led to the original suggestion that quasar jets remain highly relativistic to kpc scales; (b) At right, we plot the total radio-X-ray spectral SED for the large-scale jet of PKS 0637-752 (black points). The hard spectrum in the X-rays clearly indicates a separate spectral component, but both a synchrotron (yellow line) and an inverse Compton (black line) interpretation were plausible before the Fermi upper limits ruled out the latter for this source [14].

It has been proposed $[15,16]$ that the $\mathrm{X}$-ray emission is due to the IC/CMB process, requiring relativistic jets (bulk $\Gamma \sim 10-20$ ) oriented close to the line of sight and an electron energy distribution (EED) extending down to Lorentz factors $\gamma \sim 30$-much lower than the $\gamma \sim 10^{3}-10^{4}$ traced by GHz emission-to explain the $\mathrm{X}$-ray emission. In view of the low radiative efficiency of these electrons, reproducing the observed $\mathrm{X}$-ray flux requires high power, sometimes in excess of the Eddington luminosity of the central black hole [17-20].

An alternative interpretation of the $\mathrm{X}$-rays is synchrotron emission from high energy electrons, lying in a separate, high-energy population [21,22]. This does not require the jet to be highly relativistic or viewed at small angles, nor does it make such heavy demands on the kinetic power [17]. However, it requires extremely efficient particle acceleration, reaching at least $\gamma \sim 10^{8}$ operating in situ hundreds of kpc from the central engine.

Deciding between these very different models is difficult, as both models can explain the radio to X-ray SEDs equally well, as shown in Figure 2. However, two independent diagnostics have recently been demonstrated by our group: optical/UV polarimetry of the second component, and Fermi gamma-ray observations of the IC peak. 

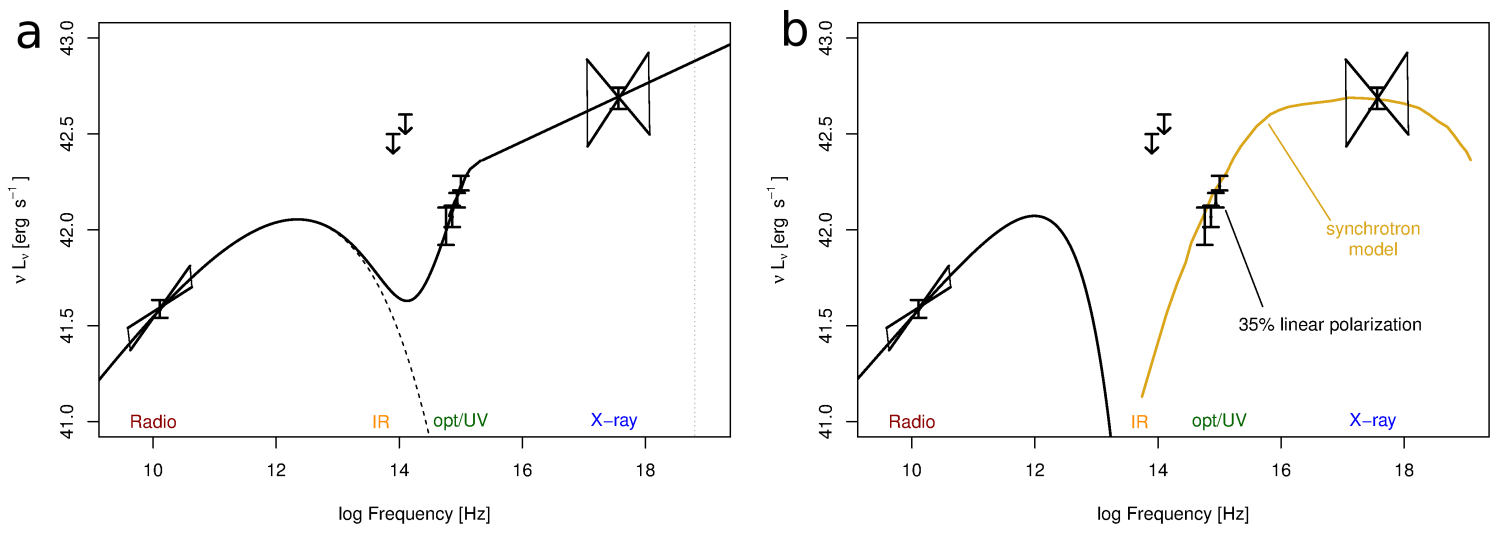

Figure 2. Both left and right figures contain identical data for the observed SED of the large-scale jet of quasar PKS 1136-135 (black points). As depicted, both an inverse Compton scattering off the cosmic microwave background (IC/CMB) model using a fast jet (a, black line), and a second-synchrotron model (b, yellow line) can reproduce the observed radio-to-X-ray SED. In the case of this particular quasar, the discovery that the UV flux was significantly polarized has shown that the synchrotron scenario is much more likely (Figures adapted from [23]).

\section{Results}

\subsection{Polarimetry Rules out IC/CMB for PKS 1136-135}

One method of discriminating between these models is the combination of spectral diagnostics with optical polarimetry. Because the CMB is unpolarized, the IC/CMB emission should also be unpolarized [24,25], for all except the very lowest energy $(\gamma \sim 1)$ particles [26-28]. Our group performed HST optical polarimetry of PKS 1136-135 [23], finding that several knots in its jet are highly polarized, with fractional polarization $\Pi_{O p t}>30 \%$. When combined with the broadband spectral shape of these regions, this is very difficult to explain via IC/CMB models, unless the scattering particles are at the lowest-energy tip of the electron energy distribution, with Lorentz factor $\gamma \sim 1$, and the jet is also very highly beamed (Doppler factor $\delta \geq 20$ ) and viewed within a few degrees of the line of sight (Figure 2). This configuration is highly unlikely, as PKS 1136-135 has a steep radio spectrum and displays neither rapid variability nor high integrated optical polarization-unlike blazars, the more typical high- $\delta$ source. Furthermore, the energetic demands of such a jet are extreme: if we require one proton per radiating lepton, the jet power must be at least $10 \times$ the Eddington luminosity of the black hole in PKS 1136-135, and such a configuration might result in Faraday depolarization in the radio [29]. Thus, the observed high polarizations strongly disfavor the possibility of the IC/CMB mechanism being responsible for the optical-to-X-ray emission in this jet.

\subsection{Fermi Rules out IC/CMB for 3C 273 and PKS 0637-752}

A $\gamma$-ray discriminant-proposed [28] and recently applied [14,30] by our group-is based on the fact that the same electrons that produce the radio to near-IR synchrotron emission must up-scatter the $\mathrm{CMB}$ photons to produce an $\mathrm{X}$-ray to $\mathrm{GeV}$ component whose amplitude depends solely on the ratio $B / \delta$, where $B$ is the magnetic field of the emitting region. The requirement that the IC/CMB emission reproduces the observed X-ray emission sets $B / \delta$, which in turn sets the level of the anticipated $\gamma$-ray emission without any freedom. Fermi observations can evaluate whether the $\mathrm{GeV}$ emission anticipated in the IC/CMB model is indeed observed. The situation is complicated by the low angular resolution of Fermi, because the steady jet emission and the variable emission from the core cannot be spatially resolved. One has to wait for periods of low nuclear activity to set deep upper limits to the jet extended emission. 
This test was recently applied to 3C 273 and PKS 0637-752 [14,30], using over six years of Fermi monitoring. As can be seen in Figure 3, the the upper limits are much lower than the IC/CMB anticipated flux level from the knots, clearly ruling out the IC/CMB model for both quasars. Even after ruling out the IC/CMB model as an explanation for the anomalously high X-ray fluxes, we still anticipate some level of IC/CMB to be produced, as it is a mandatory process. The Fermi upper limits can thus be translated to upper limits on the jet Doppler factor (adopting an equipartition magnetic field value). This results in $\delta<6.5$ for PKS 0637-752 and $\delta<7.8$ for 3C 273. Our method, therefore, can not only rule out the IC/CMB model, but it can set limits on how relativistic the large-scale jets are. These upper limits will in most cases decrease with time, as Fermi monitors the entire sky nearly continuously. We also anticipate direct detection of the IC/CMB component for some of the population we plan to study within the 10-year timespan of the full Fermi mission through August 2018.
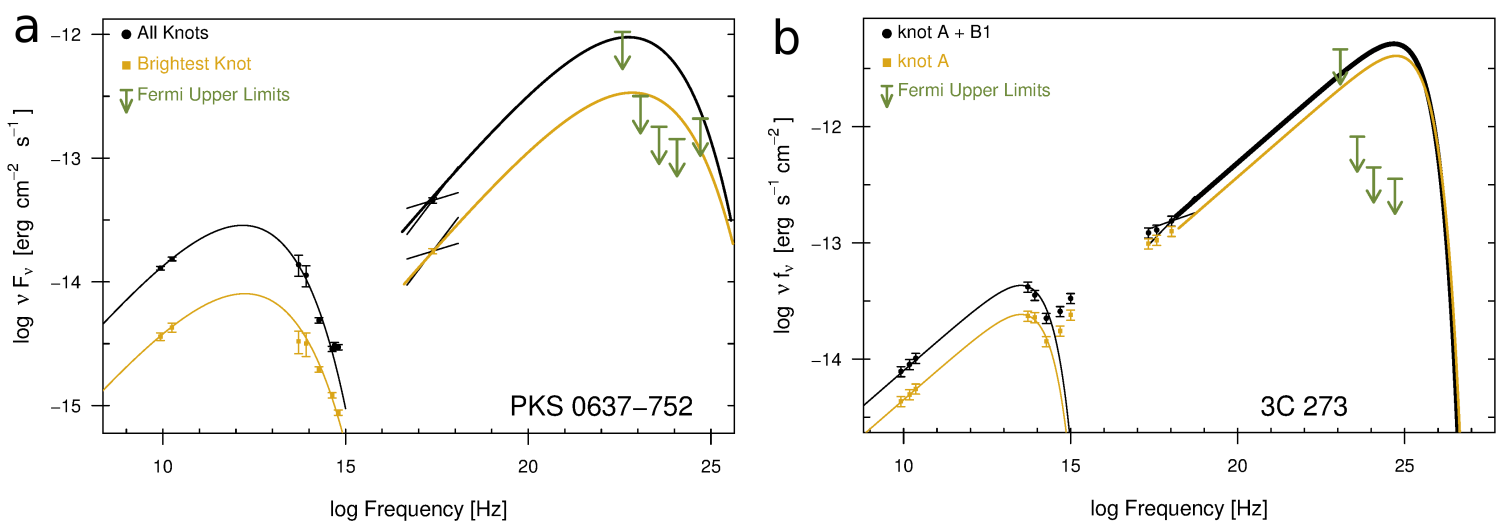

Figure 3. (a) The SED for the large-scale jet of PKS 0637-752. Data for the four brightest knots combined are shown as black points and are plotted in yellow for the single X-ray brightest knot, wk8.9; (b) The SED for the knots of 3C 273, with black points for knots A and B1 combined, and yellow for the X-ray brightest knot A only. For both plots, the Fermi 95\% upper limits are shown as dark green arrows. As can be seen, Fermi upper limits rule out IC/CMB for the X-ray emission mechanism in both sources (Figure adapted from [14]).

\section{Discussion}

\subsection{Synchrotron X-Rays from the Large-Scale Jets of Quasars}

A synchrotron origin for the $\mathrm{X}$-rays from a second population of electrons that reaches energies up to $\sim 30-100 \mathrm{TeV}$ was suggested early on (e.g., [4,31]). This interpretation is not in conflict with any of the data in hand, and further, relaxes many of the "uncomfortable" constraints of the IC/CMB model. Very small angles to the line of sight are not required, and the total jet power required is considerably less than Eddington [17], as the electron energy distribution need not be extended to very low energies. The main objection to a second synchrotron component heretofore has simply been its unexplained nature; Schwartz et al. [10] noted that there is no reason why a second population of high-energy electrons should be co-spatial with the first. However, this co-location of two very different electron populations (if correct) is obviously a very important clue to the particle acceleration mechanism in large-scale jets, of which we still know little. In addition, the multi-TeV electrons that produce the synchrotron $\mathrm{X}$-rays will also up-scatter the $\mathrm{CMB}$ to $\mathrm{TeV}$ energies, so one expects these large-scale jets to be $\mathrm{TeV}$ emitters. This is a very interesting prospect, as it has been suggested that the $\mathrm{TeV}$ photons produced in low-power $\mathrm{TeV}$ emitting blazars heat the intergalactic medium through plasma instabilities [32], and possibly explain observed discrepancies in the numbers of dwarf galaxies predicted by cosmological simulations [33]. 


\subsection{Quasar Jets: Comparable $4 \pi$ Luminosity with the Blazar Core and Bright TeV Emission}

It is usually assumed that the radiated power of quasar jets is dominated by that produced at their blazar core, the base of the jet very near the black hole that is unresolved even by very long baseline interferometry (VLBI) imaging. Certainly, the blazar observed fluxes are dominated by the core, as can be seen in Figure 4 by comparing the core and jet fluxes.
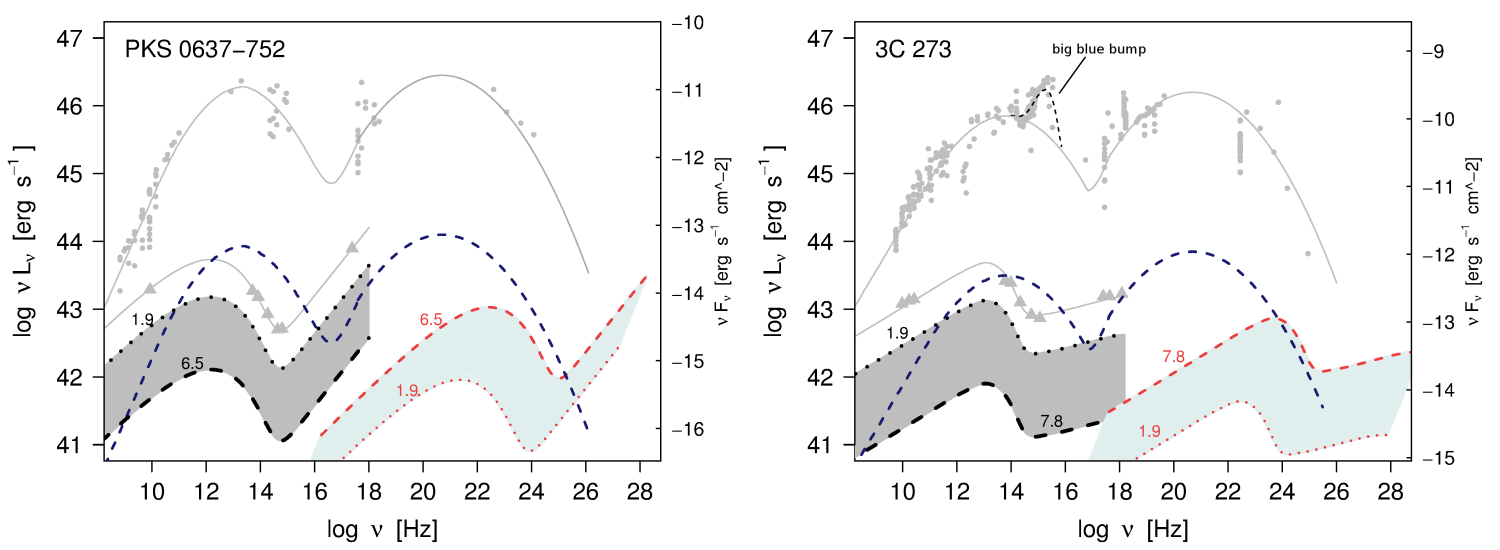

Figure 4. The following description applies to both panels, with PKS 0637-752 at left, 3C 273 at right. The core fluxes are shown as gray circles with phenomenological SED fit through the points drawn as a thin gray line. The big blue bump-visible only in 3C 373-is shown as a black dashed line and is not included in the beamed emission fits. In comparison, the total kpc-scale jet flux is shown as gray triangles. The flux scale at right only applies to solid curves in the figure. The blue dashed line is the $4 \pi$-integrated total core output. The gray zone is the range of permitted $4 \pi$-integrated output for the knots, given our current constraints on $\delta$. Finally, the light blue shaded area is the range of IC/CMB emission from the knots possible given the same $\delta$ constraints.

Our results reveal a different reality, as we now know that the large-scale jet is significantly slower than the core (see Section 2.2). VLBI superluminal motions place a lower limit on $\Gamma=15$ for the cores of both 3C 273 and PKS 0637-752 [34,35], while our Fermi limits for the large scale jets reguire $\delta<7.8$ for 3C 273 and $\delta<6.5$ for PKS 0637-752. If we correct the core fluxes for beaming by multiplying by $\delta^{-2}$ [36], we obtain the actual, $4 \pi$-integrated power output from the core (Figure 4, dark blue dashed line). To calculate the $4 \pi$-integrated luminosity of the knots, we also consider a lower limit value of $\delta=1.9$ that comes from population studies [37] and gives the dotted-line upper edge to the gray shaded area, while our $\delta$ limits from Fermi give the lower dashed-line limit. The true $4 \pi$-integrated luminosity of the knots is thus somewhere in the gray zone. Comparing the dashed-blue curve and the gray shaded area, we see that the knots are not necessarily insignificant in total output when compared to the core. Definite conclusions will require tighter constraints on the $\delta$ factors of the knots through our work. It is possible that large-scale jets contribute more than the core in the UV to X-rays, in addition to their dominance in the radio. Large-scale jets could thus also be contributors to some astrophysical backgrounds.

A further realization follows, if we assume that the $X$-rays are synchrotron in origin: the $30-100 \mathrm{TeV}$ electrons producing the X-rays will themselves upscatter the CMB to produce a GeV to TeV spectrum. The angle-integrated total power of this IC/CMB component is shown in Figure 4 as a light blue shaded area (note that the bounds in this case are flipped, see [14]). Even in the minimum $\delta=1.9$ case, these jets are already constrained to produce $4 \pi$-integrated power well in excess of $10^{41} \mathrm{erg} \mathrm{s}^{-1}$, which is the typical $4 \pi$-integrated luminosity for canonical TeV blazars (adopting an observed luminosity of $\sim 10^{43-44} \mathrm{erg} \mathrm{s}^{-1}$ and a typical $\delta \sim 20-30$ for TeV blazars). Note that because of their much lower jet $\delta$, the TeV fluxes from the knots will be approximately an order of magnitude dimmer than those 
of the $\mathrm{TeV}$ Blazars and, for nearby sources like $3 \mathrm{C} 273$, within the reach of upcoming $\mathrm{TeV}$ arrays like the CTA.

This potential source of $\mathrm{TeV}$ photons from quasar jets is important because it has been proposed that $\mathrm{TeV}$ blazars are significant contributors to the heating of the intergalactic medium (IGM; e.g., [32,38,39]). The question of the relevance of this mechanism is important, as it has been suggested to suppress the formation of late-forming dwarf galaxies [33] —an open question in the cold dark matter model for structure formation. Analytical arguments in the linear regime point to a conversion of the $\mathrm{TeV}$ photon energy-after pair production with the $\mathrm{CMB}$ - to electron-positron pairs, which, through plasma instabilities, deposit their energy with 100\% efficiency in the IGM. Contradicting this is numerical work with particle-in-cell (PIC) simulations that point to a low heating efficiency of about one percent or less [40]. These simulations, however, are confined to length scales much smaller that those of the plasma instability. It is not clear if this impasse can be solved, as both the analytical and PIC methods cannot be readily improved.

If this mechanism is important, the large-scale jets of quasars may be a far more important source of $\mathrm{TeV}$ photons than the TeV BL Lacs, and possibly the dominant class of sources for $\mathrm{TeV}$ photon heating of the IGM.

\section{Conclusions}

Apparently, the X-ray emission Chandra detects from the knots of powerful quasar jets-X-ray emission that belongs to a spectral component separate from the radio-sub-mm-IR-optical one-does not come from IC/CMB. This means that large-scale jets are not highly relativistic. This result is currently based on three sources: 3C 273 and PKS 0637-752, on which we applied our GeV diagnostic, and PKS 1136-135, a unique source where the optical is at the low energy tail of the X-ray component and manifests high optical polarization, a feature that the IC/CMB cannot reproduce. The Fermi diagnostic is being applied by our group to several large scale jets with bright X-ray knots. We hope that in the next year we will have results from these investigations, possibly confirming that the IC/CMB model is disfavored.

The question remains as to what this $\mathrm{X}$-ray emission is. This is an important question, because it will advise us of the physical conditions and processes in the jets, such as particle content, particle acceleration requirements, leptonic vs. hadronic models, $\mathrm{TeV}$ photon production, and jet power. The last two are quantities of interest for understanding feedback in galaxy cluster heating.

Acknowledgments: The authors acknowledge support from NASA/ADAP grant NNX15AE55G. E.S.P. acknowledges support from grants HST-GO-13764.001, HST-GO-13676.003, Chandra GO3-14113A and HST-GO-13114.01. E.T.M. acknowledges support from NASA/Fermi grant 81051.

Author Contributions: E.M. led the work on the Fermi method, E.P. on the polarization, and M.G. on the interpretation and implications of our findings.

Conflicts of Interest: The authors declare no conflict of interest.

\section{References}

1. Silk, J.; Mamon, G.A. The current status of galaxy formation. Res. Astron. Astrophys. 2012, 12, 917-946.

2. Fabian, A.C. Observational Evidence of Active Galactic Nuclei Feedback. Annu. Rev. Astron. Astrophys. 2012, 50, 455-489.

3. Meier, D.L.; Koide, S.; Uchida, Y. Magnetohydrodynamic Production of Relativistic Jets. Science 2001, 291, 84-92.

4. Harris, D.E.; Krawczynski, H. X-ray Emission from Extragalactic Jets. Annu. Rev. Astron. Astrophys. 2006, 44, 463-506.

5. Harris, D.E.; Sambruna, R. Relativistic Jets from Active Galactic Nuclei; Boettcher, M., Harris, D.E., Krawczynski, H., Eds.; Wiley-VCH: Weinheim, Germany, 2011; Chapter 7, pp. 184-212.

6. Perlman, E.S.; Biretta, J.A.; Sparks, W.B.; Macchetto, F.D.; Leahy, J.P. The Optical-Near-Infrared Spectrum of the M87 Jet fromHubble Space Telescope Observations. Astrophys. J. 2001, 551, 206-222. 
7. Hardcastle, M.; Birkinshaw, M.; Worrall, D.M. Chandra observations of the X-ray jet in 3C 66B. Mon. Not. R. Astron. Soc. 2001, 326, 1499-1507.

8. Perlman, E.S.; Wilson, A.S. The X-ray Emissions from the M87 Jet: Diagnostics and Physical Interpretation. Astrophys. J. 2005, 627, 140-155.

9. Chartas, G.; Worrall, D.M.; Birkinshaw, M.; Cresitello-Dittmar, M.; Cui, W.; Ghosh, K.K.; Harris, D.E.; Hooper, E.J.; Jauncey, D.L.; Kim, D.-W.; et al. The Chandra X-ray Observatory Resolves the X-ray Morphology and Spectra of a Jet in PKS 0637-752. Astrophys. J. 2000, 542, 655-666.

10. Schwartz, D.A.; Marshall, H.L.; Lovell, J.E.J.; Piner, B.G.; Tingay, S.J.; Birkinshaw, M.; Chartas, G.; Elvis, M.; Feigelson, E.D.; Ghosh, K.K.; et al. Chandra Discovery of a 100 kiloparsec X-ray Jet in PKS 0637-752. Astrophys. J. Lett. 2000, 540, L69-L72.

11. Wilson, A.S.; Young, A.J.; Shopbell, P.L. Chandra X-ray Observations of Pictor A: High-Energy Cosmic Rays in a Radio Galaxy. Astrophys. J. 2001, 547, 740-753.

12. Sambruna, R.M.; Gambill, J.K.; Maraschi, L.; Tavecchio, F.; Cerutti, R.; Cheung, C.C.; Urry, C.M.; Chartas, G. A Survey of Extended Radio Jets with Chandra and the Hubble Space Telescope. Astrophys. J. 2008, 608, 698-720.

13. Marshall, H.L.; Schwartz, D.A.; Lovell, J.E.J.; Murphy, D.W.; Worrall, D.M.; Birkinshaw, M.; Gelbord, J.M.; Perlman, E.S.; Jauncey, D.L. A Chandra Survey of Quasar Jets: First Results. Astrophys. J. Suppl. 2005, 156, 13-33.

14. Meyer, E.T.; Georganopoulos, M.; Sparks, W.B.; Perlman, E. Ruling out IC/CMB X-rays in PKS 0637-752 and the Implications for TeV Emission from Large-scale Quasar Jets. Astrophys. J. 2015, 805, 154.

15. Tavecchio, F.; Maraschi, L.; Sambruna, R.M.; Urry, C.M. The X-ray Jet of PKS 0637-752: Inverse Compton Radiation from the Cosmic Microwave Background? Astrophys. J. 2000, 544, L23-L26.

16. Celotti, A.; Ghisellini, G.; Chiaberge, M. Large-scale jets in active galactic nuclei: Multiwavelength mapping. Mon. Not. R. Astron. Soc. 2001, 321, L1-L5.

17. Dermer, C.D.; Atoyan, A. Nonthermal Radiation Processes in X-ray Jets. Astrophys. J. Lett. 2004, 611, L9-L12.

18. Mehta, K.T.; Georganopoulos, M.; Perlman, E.S.; Padgett, C.A.; Chartas, G. Hubble Space Telescope Observations of the Quasar PKS 0637-752: Equipartition Electron-Proton Jet from the Most Complete Spectral Coverage to Date. Astrophys. J. 2009, 690, 1706-1714.

19. Uchiyama, Y.; Urry, C.M.; Cheung, C.C.; Jester, S.; van Duyne, J.; Coppi, P.; Sambruna, R.M.; Takahashi, T.; Tavecchio, F.; Maraschi, L. Shedding New Light on the 3C 273 Jet with the Spitzer Space Telescope. Astrophys. J. 2006, 648, 910-921.

20. Dermer, C.D.; Finke, J.D.; Krug, H.; Böttcher, M. Gamma-Ray Studies of Blazars: Synchro-Compton Analysis of Flat Spectrum Radio Quasars. Astrophys. J. 2009, 692, 32-46.

21. Jester, S.; Harris, D.E.; Marshall, H.L.; Meisenheimer, K. New Chandra Observations of the Jet in 3C 273. I. Softer X-ray than Radio Spectra and the X-ray Emission Mechanism. Astrophys. J. 2006, 648, 900-909.

22. Hardcastle, M.J. Testing the beamed inverse-Compton model for jet X-ray emission: velocity structure and deceleration. Mon. Not. R. Astron. Soc. 2006, 366, 1465-1474.

23. Cara, M.; Perlman, E.S.; Uchiyama, Y.; Cheung, C.C.; Coppi, P.S.; Georganopoulos, M.; Worrall, D.M.; Birkinshaw, M.; Sparks, W. B.; Marshall, H.L.; et al. Polarimetry and the High-energy Emission Mechanisms in Quasar Jets: The Case of PKS 1136-135. Astrophys. J. 2013, 773, 186-198.

24. Uchiyama, Y.; Urry, C.M.; Coppi, P.; van Duyne, J.; Cheung, C.C.; Sambruna, R.M.; Takahashi, T.; Tavecchio, F.; Maraschi, L. An Infrared Study of the Large-Scale Jet in Quasar PKS 1136-135. Astrophys. J. 2007, 661, 719-727.

25. Krawczynski, H. The Polarization Properties of Inverse Compton Emission and Implications for Blazar Observations with the GEMS X-ray Polarimeter. Astrophys. J. 2012, 774, 30-43.

26. Begelman, M.C.; Sikora, M. Inverse Compton scattering of ambient radiation by a cold relativistic jet-A source of beamed, polarized continuum in blazars? Astrophys. J. 1987, 322, 650-661.

27. Poutanen, J.; Vilhu, O. Compton scattering of polarized light in two-phase accretion discs. Astron. Astrophys. 1993, 275, 337-344.

28. Georganopoulos, M.; Perlman, E.S.; Kazanas, D.; McEnery, J. Quasar X-ray Jets: Gamma-ray Diagnostics of the Synchrotron and Inverse Compton Hypotheses: The Case of 3C 273. Astrophys. J. Lett. 2006, 653, L5-L8.

29. Jones, T.W.; Odell, S.L. Transfer of polarized radiation in self-absorbed synchrotron sources. II. Treatment of inhomogeneous media and calculation of emergent polarization. Astrophys. J. 1977, 215, 236-246. 
30. Meyer, E.T.; Georganopoulos, M. Fermi rules out the Inverse-Compton/CMB model for the Large-Scale Jet X-ray emission of 3C 273. Astrophys. J. 2014, 780, 27.

31. Hardcastle, M.J.; Harris, D.E.; Worrall, D.M.; Birkinshaw, M. The Origins of X-ray Emission from the Hot Spots of FR II Radio Sources. Astrophys. J. 2004, 612, 729-748.

32. Broderick, A.E.; Chang, P.; Pfrommer, C. The Cosmological Impact of Luminous TeV Blazars. I. Implications of Plasma Instabilities for the Intergalactic Magnetic Field and Extragalactic Gamma-Ray Backgroun. Astrophys. J. 2012, 752, 22.

33. Pfrommer, C.; Chang, P.; Broderick, A.E. The Cosmological Impact of Luminous TeV Blazars. III. Implications for Galaxy Clusters and the Formation of Dwarf Galaxies. Astrophys. J. 2012, 752, 24.

34. Lister, M.L.; Aller, M.F.; Aller, H.D.; Homan, D.C.; Kellermann, K.I.; Kovalev, Y.Y.; Pushkarev, A.B.; Richards, J.L.; Ros, E.; Savolainen, T. MOJAVE. X. Parsec-scale Jet Orientation Variations and Superluminal Motion in Active Galactic Nuclei. Astrophys. J. 2013, 146, 120.

35. Edwards, P.G.; Piner, B.G.; Tingay, S.J.; Lovell, J.E.J.; Kataoka, J.; Ojha, R.; Murata, Y. The Parsec-Scale Jet of PKS 0637-752. Publ. Astron. Soc. Jpn. 2006, 58, 233-241.

36. Ghisellini, G.; Tavecchio, F. Compton rockets and the minimum power of relativistic jets. Mon. Not. R. Astron. Soc. 2010, 409, L79-L83.

37. Arshakian, T.G.; Longair, M.S. On the jet speeds of classical double radio sources. Mon. Not. R. Astron. Soc. 2004, 351, 727-732.

38. Chang, P.; Broderick, A.E.; Pfrommer, C. The Cosmological Impact of Luminous TeV Blazars. II. Rewriting the Thermal History of the Intergalactic Medium. Astrophys. J. 2012, 752, 23.

39. Lamberts, A.; Chang, P.; Pfrommer, C.; Puchwein, E.; Broderick, A.E.; Shalaby, M. Patchy Blazar Heating: Diversifying the Thermal History of the Intergalactic Medium. Astrophys. J. 2015, 811, L19.

40. Sironi, L.; Giannios, D. Relativistic Pair Beams from TeV Blazars: A Source of Reprocessed GeV Emission rather than Intergalactic Heating. Astrophys. J. 2014, 787, 49.

(C) 2016 by the authors; licensee MDPI, Basel, Switzerland. This article is an open access article distributed under the terms and conditions of the Creative Commons Attribution (CC-BY) license (http://creativecommons.org/licenses/by/4.0/). 